\title{
A method for restricting stimuli to the frontal or lateral visual field of each eye separately in pigeon'
}

This report describes a method of restricting visual stimuli either to the frontal or to the lateral visual field in pigeons, using goggles and colored filters. This technique permits the sequential or simultaneous presentation of discriminative stimuli to each eye separately for the study of inter-hemispheric transfer of visual information. Behavioral data are presented to demonstrate the adequacy of the method.

The simultaneous presentation of visual stimuli to each eye separately has been accomplished by use of a device in which each eye is covered either with oppositely polarized filters (Trevarthan, 1962) or color filters with no overlap in spectral transmittance (Gazzaniga, 1966; Mello, 1967; Robinson \& Voneida, 1964). The discriminative stimuli are constructed of the same polarized or colored material as the goggle filter. Each filter transmits only the discriminative stimulus pattern constructed of the same color or polarized filter. The use of polarized filters is contraindicated in a free-ranging pigeon since the pigeon could tilt its head so that the eye covered with a vertically polarized filter could detect a stimulus projected through a horizontally polarized filter. Method

A modification of the goggle originally described by Catania (1963) is shown in Fig. 1. ${ }^{3}$ Goggles are attached to the pigeon's scalp with a fast -setting dental acrylic. The basic goggle(Fig. 1a) is constructed from brass tubing $1 / 32$ in. thick with a $9 / 16$ in. inner diameter'and a $5 / 8 \mathrm{ln}$. outer diameter. The tubing is cut at a $45^{\circ}$ angle leaving a $1 / 16$ in. 1and. The longest portion of the goggle measures $11 / 16$ in. The addition of a machine nut $(2-56)$, filed flat on one surface and soldered directly to the side of the goggle (Fig. 1b), makes it possible to affix any type of goggle cover to the goggle with a 2-56 machine screw.

A second modification of the basic goggle is the rectangular hole ( $9 \mathrm{~mm} \times 7 \mathrm{~mm}$ ) in the side which exposes the pigeon's lateral visual field (Fig. 1c). This side aperture permits presentation of laterally placed visual stimuli to the lateral visual field. Filters can be attached to this surface permanently with dental acrylic or temporarily with electrical Scotch tape.

In order to restrict discriminative stimuli to each eye separately, the goggles were covered with red and blue filters which were cemented between two oval sheets of $0.040 \mathrm{in}$. clear Plexiglas, with Dupont Duco cement. The spectral absorption of the Plexiglas was analyzed with a Beckman DU Spectrophotometer. The absorbence $\left(\log _{10} \mathrm{I} / \mathrm{I}\right)$ values obtained were comparable across the visible spectrum. The red (No. 29) and blue (No. 45) Wratten filters selected have no overlap in spectral transmittance. The dominant wavelength of Wratten Filter No. 45 is $\mathbf{4 8 6 . 6}$ with a range of transmittance between 440 and $540 \mathrm{~m} \mu$. The dominant wavelength of Wratten Filter No. 29 is 632.7 with a transmittance range between 610 and $700 \mathrm{~m} \mu$. Discriminative stimuli were constructed from the same Wratten filters and projected through an in-line digital-display device obtatned from Industrial Electronic Engineers, Inc. Since there is no overlap in the spectral transmittance range of these red and blue Wratten filters, only a red stimulus can be detected through the red filter and a blue stimulus appears black to the human observer.
In order to insure that pigeons could not detect a blue stimulus while wearing red goggles on the basis of any other cues, pigeons were trained to discriminate a blue horizontal line ( $S-$ ) from a blue vertical line ( $\mathrm{S}+$ ) while wearing blue goggles. Subsequently, discrimination performance was tested under the following conditions: (1) blue goggles and blue discriminative stimuli (as during training); (2) blue goggles and red discriminative stimuli; (3) red goggles and red discriminative stimuli; (4) red goggles and blue discriminative stimuli. In each instance, the discriminative stimuli used during testing were the same as those used during training.

Pigeons were trained for 10 sessions on a chain DRO VI 1 schedule of reinforcement. The details of the training procedure and apparatus have been more fully described in previous reports (cf., Mello, 1966). When the positive stimulus (S+) was displayed upon the response key, the first peck after a variable interval of time (averaging $1 \mathrm{~min}$.) resulted in access to grain for $6 \mathrm{sec}$. Fivemin. presentations of $S+$ alternated with presentations of the negative stimulus ( $S-$ ) during which no reinforcements were available. Termination of the S- was contingent upon the pigeon's withholding pecking for a period of $1 \mathrm{~min}$. in the final reinforcement schedule (chain DRO VI 1 ).

Tests were run under extinction conditions and the discriminative stimuli (S+ and S-) were presented successively for $1 \mathrm{~min}$. periods. The test sequence was divided into four consecutive blocks of eight stimulus presentations. During the first and third block of eight stimuli, the color filters over the discriminative stimuli and over the pigeons' goggles were different. During the second and fourth block of eight stimulus presentations, the color filters over the stimuli and the goggles were the same. This sequence of alternate 8-min. periods was used to distribute the possible effects of no reinforcement on the response rate equally between the two conditions.

\section{Results and Discussion}

The data presented in Table 1 show that trained pigeons were able to discriminate $S+$ from $S-$ only under conditions where the same filters covered the goggles and the discriminative stimuli. When pigeons (RB-83 and YB-4) wearing blue goggles were presented with red discriminative stimuli or the converse (RB-82), there were no responses or the distribution of re-

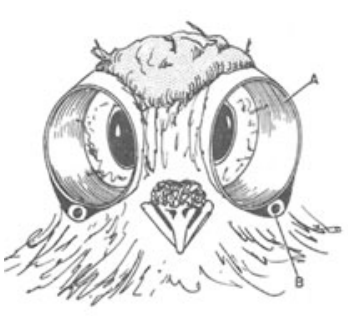

FRONTAL VISUAL FIELD

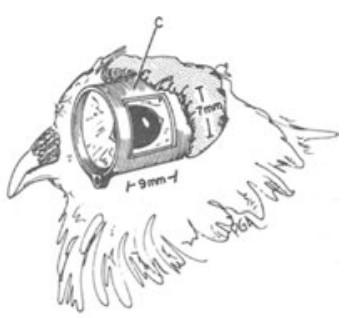

LATERAL VISUAL FIEL
Fig. 1. Goggles to permit restriction of visual stimuli to the frontal or lateral visual field of each eye separately. (A) the basic goggle cut from $1 / 32$ in. brass tubing; (B) a machine nut (2-56) soldered to the goggle to which a cover can be attached with a machine screw (2-56, 1/8 in. round head); $(C)$ an aperture to permit stimulation of the lateral visual field. 
Table 1. Comparison of responses during four sequential extinction tests when the colored filters over the pigeons goggles and the discriminative stimuli were the same and different. Pigeons were trained to discriminate between a vertical $\left(S_{+}\right)$and a horizontal $\left(S_{-}\right)$rectangle. $S_{+}$is the stimulus associated with reinforcement and $\mathbf{S -}$ is the stimulus never associated with reinforcement for responding.

\begin{tabular}{|c|c|c|c|c|c|c|c|c|c|c|c|c|}
\hline \multirow{3}{*}{$\begin{array}{l}\text { Goggle and } \\
\text { Stimulus Filter } \\
\text { Pigeon }\end{array}$} & \multicolumn{3}{|c|}{ Different } & \multicolumn{3}{|c|}{ Same } & \multicolumn{3}{|c|}{ Different } & \multicolumn{3}{|c|}{ Same } \\
\hline & $S_{+}$ & S- & $S_{+}$ & $S_{+}$ & S- & $s_{+}$ & $\mathrm{S}_{+}$ & $S$ & $S_{+}$ & $S_{+}$ & S- & $S_{+}$ \\
\hline & & & $S+\& S$ & & & $\overline{S+\& S}$ & & & $\overline{S+\& S}$ & & & $S+\& S$ \\
\hline $\begin{array}{l}\text { RB- } 82 \\
\text { (Red Goggles) }\end{array}$ & 0 & 0 & $(--)$ & 179 & 40 & $(82 \%)$ & 0 & 0 & $(--)$ & 109 & 0 & $(100 \%)$ \\
\hline $\begin{array}{l}\text { RB-83 } \\
\text { (Blue Goggles) }\end{array}$ & 27 & 24 & $(52 \%)$ & 170 & 6 & $(97 \%)$ & 29 & 16 & $(64 \%)$ & 104 & 14 & $(88 \%)$ \\
\hline $\begin{array}{l}\text { YB-4 } \\
\text { (Blue Goggles) }\end{array}$ & 13 & 7 & $(65 \%)$ & 225 & 15 & $(94 \%)$ & 0 & 0 & $(--)$ & 52 & 0 & $(100 \%)$ \\
\hline
\end{tabular}

sponses to $S+$ and $S-$ were close to chance. These behavioral data are consistent with the expected result since there is no overlap in the spectral transmittance range of the red and blue filters. However, one pigeon was run on a regular training session with red discriminative stimuli, while wearing blue goggles, in order to insure that discrimination did not occur under conditions of differential reinforcement.

A comparison of the cumulative records of the performance of RB-83 with different filters over the goggles and stimuli and with the same filters over goggles and stimuli, on the tenth day of training, is presented in Fig. 2. It is apparent that although its performance indicated a good discrimination between S+ and S- on Day 10, these stimuli exerted no control over response behavior when the goggle filters were different from the stimulus filters even though the

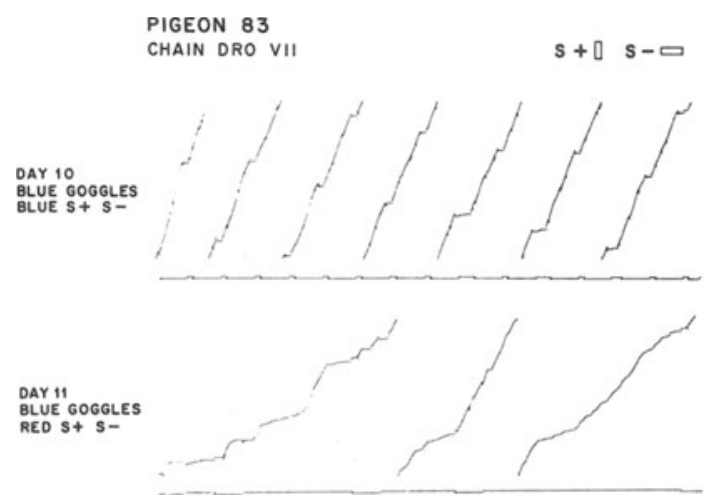

Fig. 2. Cumulative records of responses during discrimination training using a two-component schedule of reinforcement (chain DRO VI 1). The upper trace in each record represents cumulative responses as a function of time and each downward deflection of this pen indicates the occurrence of a grain reinforcement. The lower trace in each record indicates the schedule of reinforcement in effect: Where the pen is elevated, the stimulus was a vertical line $\left(S_{+}\right)$and responses were intermittently reinforced; where the pen is depressed, the stimulus was a horizontal line (S-) and responses were not reinforced. Any response during $\mathbf{S}-$ prolonged the duration of $\mathbf{S}_{-}$, and return to $\mathbf{S}_{+}$was contingent upon zero responding for a period of $1 \mathrm{~min}$. The $S_{+}$is present in the first segment of each record. schedule of reinforcement was identical. This performance disruption indicates that the blue goggle filters effectively prevented visual stimulation by the red stimuli; i.e., the pigeon was unable to perform a previously learned discrimination on the basis of other, uncontrolled cues.

This technique provides a method to train pigeons on separate discrimination problems with each eye, either sequentially or concurrently, merely by manipulating the color of the filter over the goggles and the color of the discriminative stimuli. Finally, this procedure makes it possible to examine the capacity of the pigeon to compare two stimuli, each of which are presented separately to one eye with respect to their similarity or difference in size or orientation (cf., Mello, 1967).

\section{References}

Catania, A.C. Techniques for the control of monocular and binocular viewing in pigeon. J. exp. Anal. Behav., 1963, 6, 627. Gazzaniga, M. A. Interhemispberic cuing systems remaining after section of neo-cortical commisures in monkeys. Exp. Neurol., $1966,16,28$.

Mello, N. K. Interocular generalization: a study of mirror-image reversal following monocular discrimination training in pigeon. J. exp. Anal. Behav., 1966, 9, 11.

Mello, N. K. Inter-hemispheric comparison of stimuli simultaneously presented to the frontal visual field of pigeon. Natute, 1967, (in press).

Robinson, J. S., \& Voneida, T. J. Central cross-integration of visual inputs presented simultaneously to the separate eyes. J. comp. physiol. Psychol., 1964, 57, 22.

Trevarthan, C. B. Double visual learning in split-brain monkeys. Science, 1962, 136, 258.

\section{Notes}

1. This research was supported by grant No. NB 05004 from the National Institute of Neurological Diseases and Blindness, and by the Air Force Office of Scientific Research, Office of Aerospace Research, United States Air Force, under AFOSR grant No. 554-65.

2. Present address: NIMH, National Center for Prevention and Control of Alcoholism, 5454 Wisconsin Avenue, Chevy Chase, Maryland 20023. Reprints may be obtained from the author at this address.

3. Goggles were obtained from Mr. George Silkwood, Department of Psychology, William James Hall, Harvard University, Cambridge, Massachusetts, at $\$ 5.00$ per pair. 